\title{
Potential regulatory molecules in the human trabecular meshwork of patients with glaucoma: Immunohistochemical profile of a number of inflammatory cytokines
}

\author{
SAMANTA TAURONE $^{1}$, GUIDO RIPANDELLI $^{2}$, ELENA PACELLA ${ }^{1}$, ENRICA BIANCHI $^{1}$, \\ ANDREA MARIA PLATEROTI ${ }^{1}$, STEFANIA DE VITO ${ }^{3}$, PASQUALE PLATEROTI ${ }^{1}$, \\ FRANCESCA ROMANA GRIPPAUDO ${ }^{4}$, CARLO CAVALLOTTI $^{3}$ and MARCO ARTICO ${ }^{1}$
}

\author{
${ }^{1}$ Department of Sensory Organs, Sapienza University of Rome, Rome 00161; ${ }^{2}$ G.B. Bietti Eye Foundation-IRCCS, \\ Rome 00198; ${ }^{3}$ Department of Anatomical, Histological, Medico-legal and Locomotor System Sciences, Rome 00161; \\ ${ }^{4}$ Department of Neurosciences, Mental Health and Sensory Organs, II Faculty of Medicine and Surgery,
}

Sapienza University of Rome, Rome 00189, Italy

Received October 23, 2013; Accepted May 2, 2014

DOI: $10.3892 / \mathrm{mmr} .2014 .2772$

\begin{abstract}
Glaucoma occurs when there are imbalances between the production and the drainage of the eye liquid. The vast majority of the aqueous humor leaves the eye through the trabecular meshwork (TM). The cause of hypertonicity may be due to an alteration in the thickness of the TM. In the majority of cases the molecular changes that determine primary open-angle glaucoma (POAG) are unclear. However, it has been hypothesized that the significant increase in the extracellular matrix (ECM) of the fibrillary bands in the TM is associated with possible inflammatory conditions. In this study the tissue distribution of interleukin (IL)-6, IL-1 $\beta$, transforming growth factor- $\beta 1$ (TGF- $\beta 1)$, vascular endothelial growth factor (VEGF) and tumor necrosis factor $\alpha$ (TNF- $\alpha$ ) was analyzed in TM samples from patients with POAG by immunohistochemistry. Seven specimens from patients with POAG and three control tissues were analyzed by immunohistochemistry using specific antibodies against these cytokines. Morphological changes in the TM, such as increased cell content, macrophages, fibrosis and accumulation of neutrophils, were observed by transmission electron microscopy. In human TM tissues, an evident immunoreactivity for IL-6, IL-1 $\beta$ and TNF- $\alpha$ was observed in patients with POAG when compared with the control subjects, indicating that these cytokines may be correlated with disease activity. $\mathrm{TM}$ endothelial cells secrete a number of factors and cytokines
\end{abstract}

Correspondence to: Dr Enrica Bianchi, Department of Sensory Organs, Sapienza University of Rome, V. le del Policlinico 155, Rome 00161, Italy

E-mail: enrica.bianchi@uniroma1.it; b.enrica@yahoo.it

Key words: primary open-angle glaucoma, immunohistochemistry, intraocular pressure, interleukin-6, interleukin-1 $\beta$, tumor necrosis factor $\alpha$, tumor growth factor $\beta 1$, vascular endothelial growth factor that modulate the functions of the cells and the ECM of the conventional outflow pathway. In the TM in glaucoma, macrophages produce cytokines, including IL- $6, \mathrm{IL}-1 \beta$ and TNF- $\alpha$, leading to an acute inflammatory response and recruitment of other immune cells, including $\mathrm{T}$ lymphocytes. In addition, TGF- $\beta 1$ regulates and induces the expression of IL- 6 in TM that indirectly induces angiogenesis by stimulating VEGF expression. The present results support previous evidence that suggests that growth factors and cytokines can induce ECM remodelling and alter cytoskeletal interactions in the TM.

\section{Introduction}

Glaucoma is the second leading cause of blindness worldwide (1). During its pathogenesis, optic nerve axons are continuously damaged at the optic nerve head, a process that leads to axonal degeneration, apoptosis of retinal ganglion cells and, finally, characteristic defects in the visual field of the affected patients $(2,3)$. Glaucoma occurs in the presence of imbalances between the production and drainage of the aqueous humor (AH), due to obstruction of the outflow pathways of the trabecular meshwork (TM).

The most critical risk factor for the progression of glaucomatous optic nerve damage has been identified in several studies as intraocular pressure (IOP) $(4,5)$. IOP is generated and maintained by the AH circulatory system in the anterior eye (4). AH is actively secreted by the epithelial layers of the ciliary body into the posterior chamber of the eye and leaves the eye through the iridocorneal angle and Schlemm's canal (SC) (5). The vast majority of the AH leaves the eye through the TM, across the SC and finally enters the general circulation. When AH has passed through the trabecular outflow pathways, it drains into the episcleral venous system (5). The TM pathways are critical in providing resistance to AH outflow. The decisive factor causing hypertonicity may be an alteration in the thickness of the TM. An increase in the thickness of the TM may alter the drainage of AH due to the presence of higher resistance. A thickened TM may be correlated with the 
lamellar deposition of collagen and the accumulation of amorphous material that reduces the interlamellar spaces and the lumen of juxtacanalicular pathways. IOP builds up in response to this resistance until it is high enough to allow $\mathrm{AH}$ to flow across the TM into SC (6).

The TM consists of three regions that differ in structure: The inner uveal meshwork, the deeper corneoscleral meshwork and the juxtacanalicular tissue (JCT), or cribriform region, which is localized directly adjacent to the inner endothelial wall of the SC. The uveal meshwork, which originates from the anterior chamber of the ciliary body, consists of one to three layers of trabecular beams or lamellae. The corneoscleral meshwork forms 8-15 trabecular layers, which are thicker compared with those of the uveal TM and originate from the scleral spur. The JCT, which is localized directly in the endothelial lining of the SC, is the smallest part of the TM with a thickness of only 2-20 $\mu \mathrm{m}$. The JCT does not form trabecular lamellae or connective tissue beams, but rather represents a typical loose connective tissue with 2-5 layers of scattered cells that are embedded in a loosely arranged fibrillar ECM (5).

In primary open-angle glaucoma (POAG), there is a gradual obstruction of the outflow channels, and in the more rare narrow-angle glaucoma, drainage systems suddenly become blocked. The increased pressure within the eye compresses the small blood vessels and the fibers of the optic nerve, causing a progressive loss of vision that may result in blindness.

In the majority of cases the molecular changes that determine POAG are unclear. However, it is hypothesized that it is due to the significant increase of fibrillary bands of the ECM in the TM. This hypothesis is supported by the observation that treatment with metalloproteinases degrades components of the ECM and leads to an improvement in the AH outflow.

The TM cells have contractile properties, thus an increased tone of the TM modifies the resistance to outflow. Consequently, increased contraction of the TM may lead to increased rigidity. In fact, the destruction of actin filaments diminishes resistance to AH outflow (7).

The pathological processes that determine resistance to AH outflow in the TM, and consequently increase of IOP, are constituted by an acute inflammatory phase, in which there is infiltration of inflammatory cells and upregulation of various cytokines; a proliferative phase in which there is migration of fibroblasts and vascular endothelial cells from the surrounding tissues, and transformation of fibroblasts into myofibroblasts; and a remodelling phase, which leads to the formation of a fibrous scar (8).

In addition, numerous fibroangiogenic growth factors have been implicated in POAG pathogenesis, including tumor necrosis factor- $\alpha(\mathrm{TNF}-\alpha)$ and transforming growth factor- $\beta 1$ (TGF- $\beta 1$ ).

Angiogenesis is defined as the formation of novel blood vessels from pre-existing vasculature and underlies a large number of physiological processes, including growth and differentiation, wound healing and pathological conditions, such as neoplasia and complicated ocular diseases, which result in severe loss of vision.

The processes of vascularization involve the activation of cell-derived angiogenic factors and the appropriate synthesis of extracellular matrix (ECM) components, required for anchorage of the endothelium. One of the most potent and specific angiogenic factors is vascular endothelial growth factor (VEGF) (9). Additionally, pro-inflammatory cytokines, including interleukin (IL)-6 and IL-1 $\beta$, may be involved in mediating the inflammation associated with different ocular diseases.

IL-6, IL-1 $\beta$ and TNF- $\alpha$ have numerous characteristics similar to VEGF and are also reported to be induced by hypoxia. Increased IL-6 in aqueous humor was noted in intraocular inflammatory conditions, such as uveitis and ophthalmitis. Furthermore, Cohen et al (10) demonstrated that pro-inflammatory cytokines regulate VEGF expression. In previous studies, increased levels of VEGF were reported in samples of vitreous humour and $\mathrm{AH}$ of patients with POAG (11). In the present study the concentrations of IL-6, IL- $1 \beta$, TGF- $\beta 1$, VEGF and TNF- $\alpha$ were measured in TM samples taken from patients with POAG.

\section{Materials and methods}

Ethical considerations. A total of seven adult patients affected by POAG who had undergone a trabeculectomy were studied, together with three female autoptic specimens (from patients without ocular and/or inflammatory pathologies) harvested as control cases. The patients, aged between 45 and 69 years, were females $(n=2)$ and males $(n=5)$. The uncorrected baseline IOP in POAG eyes ranged from 26 to $30 \mathrm{~mm} \mathrm{Hg}$. The protocol and informed written consent forms were approved by the Ethical Committee of the Sapienza University of Rome (Rome, Italy) and G.B. Bietti Eye Foundation (Rome, Italy). Prior to signing the informed consent form, the patients were informed about the study in detail by a physician providing them with ample time to ask possible questions. The study was conducted in accordance with the Declaration of Helsinki. Each clinical unit selected specimens and assigned a progressive number to each sample followed by a letter indicative of the participating unit. For each case, a report was prepared indicating the age and gender of the patients and their general clinical characteristics. The control morphological sections were stained with hematoxylin and eosin. The following factors were investigated: VEGF, TGF- $\beta 1$, IL-1 $\beta$, IL-6 and TNF- $\alpha$.

Immunohistochemical analysis. The samples were washed in phosphate-buffered saline (PBS), fixed in $10 \%$ formalin and embedded in paraffin according to a standard procedure. The method employed for immunohistochemical tests was the ABC/HRP technique (avidin complexed with biotinylated peroxidase). Serial $3-\mu \mathrm{m}$ thick sections were cut using a rotative microtome, mounted on gelatin-coated slides and processed for immunohistochemistry. These sections were deparaffinized in xylene, dehydrated, immersed in citrate buffer ( $\mathrm{pH}$ 6.0) and subjected to microwave irradiation twice for 5 mins. Subsequently, all the sections were treated for 30 mins with $0.3 \%$ hydrogen peroxide in methanol to quench endogenous peroxidase activity. In order to prevent non-specific binding, the slides were incubated in 3\% normal goat serum in PBS for $30 \mathrm{mins}$ at room temperature. The slides were incubated overnight at $4^{\circ} \mathrm{C}$ with the following antibodies: i) Rabbit anti-IL-1 $\beta$ polyclonal antibody; ii) rabbit anti-IL-6 polyclonal antibody; iii) mouse anti-TNF- $\alpha$ monoclonal antibody; iv) mouse anti-VEGF monoclonal antibody; 
and v) rabbit anti-TGF- $\beta 1$ polyclonal antibody (all Santa Cruz Biotechnology, Santa Cruz, CA, USA). Optimal antisera dilutions and incubation times were assessed in a series of preliminary experiments. Following exposure to the primary antibodies, the slides were rinsed twice in phosphate buffer and incubated for $1 \mathrm{~h}$ at room temperature with the appropriate secondary biotinylated goat anti-mouse or anti-rabbit IgG antibodies (Vectastain Elite ABC Kit Standard* PK-100; Vector Laboratories Burlingame, CA, USA). Following a further wash with phosphate buffer, the slides were treated with $0.05 \%$ 3,3-diaminobenzidine and $0.1 \% \mathrm{H}_{2} \mathrm{O}_{2}$. Finally, the sections were counterstained with Mayer's hematoxylin and observed using a light microscope (Zeiss photomicroscope III; Carl Zeiss AG, Jena, Germany). Negative control experiments were performed by omitting the primary antibody; by substituting the primary antibody with an equivalent quantity of non-specific immunoglobulin; or by pre-incubating the primary antibody with the specific blocking peptide. The staining assessment was conducted by two experienced histologists. The intensity of the immune reaction was assessed microdensitometrically using an IAS 2000 image analyzer (Delta Sistemi, Rome, Italy) connected via a TV camera to the microscope. The system was calibrated taking zero as the background obtained in sections exposed to non-immune serum. A total of $10100-\mu \mathrm{m}^{2}$ areas were delineated in each section using a measuring diaphragm. The quantitative data regarding the intensity of immune staining were statistically analyzed using analysis of variance (ANOVA) followed by Duncan's multiple range as a post hoc test.

Transmission electron microscopy (TEM). A standard trabeculectomy was performed. A limbus-based conjunctival flap was raised, and a $4 \times 4-\mathrm{mm}$ half-thickness scleral flap was dissected. A window was formed by removing a block of trabecular tissue $(\sim 1 \times 2 \mathrm{~mm})$. The trabecular tissues were fixed immediately in buffer containing $2 \%$ glutaraldehyde for $2 \mathrm{~h}$, washed, postfixed in buffer with $2 \%$ osmium tetroxide for $2 \mathrm{~h}$, dehydrated and embedded in araldite. Ultrathin sections were cut using a Reichert Ultra-microtome (Reichert Technologies, Munich, Germany). These sections were counterstained with uranyl acetate and lead citrate and observed under a Zeiss EM 109 electron microscope (Carl Zeiss AG).

Statistical analysis. Quantitative data related to immune staining were analyzed statistically by ANOVA followed by Duncan's multiple range as a post hoc test. The comparison of the expression levels of VEGF, TGF- $\beta$, TNF- $\alpha$, IL- $1 \beta$ and IL-6 in the TM from glaucomatous eyes and eyes of control patients was performed by a t-test. Statistical analyses were performed using the SPSS statistical software package version 12.0 (SPSS Inc., Chicago, IL, USA). P $<0.05$ was used to indicate a statistically significant difference.

\section{Results}

TEM. The morphological changes that occurred in the TM of glaucomatous eyes were analyzed using TEM (Figs. 1 and 2). Changes, such as increased cellular content, macrophages, fibrosis and accumulation of neutrophils were evaluated.

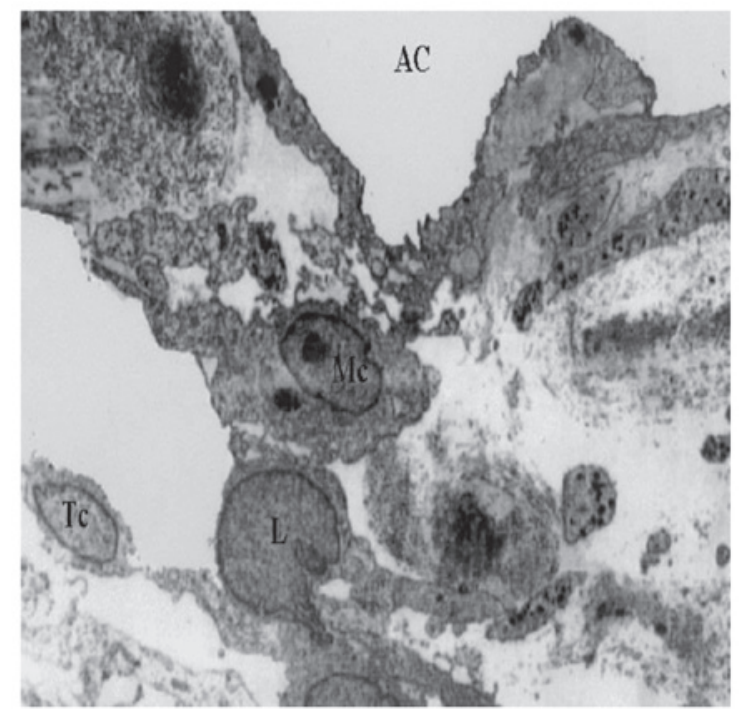

Figure 1. Transition electron microscopy micrograph of the innermost section of the uveal meshwork. AC, anterior camber; M, macrophage; L, lymphocyte; Mc, melanocyte; TC, trabecular cell. Magnification, x5, 600.

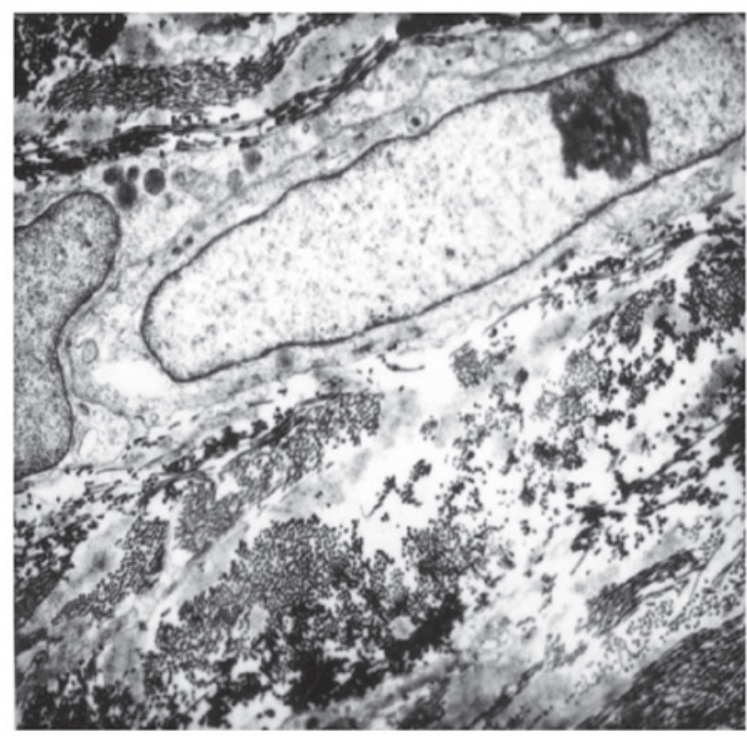

Figure 2. Transition electron microscopy micrograph. Trabecular cell with plaques of microfilaments attached to the plasma membrane and a mononuclear cell. Magnification, x5,000.

In all the cases, proliferation of fibrous connective tissue was observed at the inner wall of SC. Accumulation of collagen fibers, irregular striations and agglomerations of microfibrillar material with the structure of collagen microfibrils were observed. The AH outflow pathways of the inner TM were obstructed by cellular fragments.

The outer TM was characterized by deposition of extracellular material and collagen, presence of degenerative cells and collapse of the trabecular lamellae.

TEM images of the innermost section of the uveal meshwork (Fig. 1) revealed distribution of macrophages, lymphocytes and melanocytes. In the anterior uveal compartment the connective tissue possessed a rich population of resident tissue macrophages. Inflammatory changes, 
Table I. Expression levels of IL-6, IL-1 $\beta$, TNF- $\alpha$, TGF- $\beta 1$ and VEGF in POAG and control specimens, and respective levels of statistical significance (t-test).

\begin{tabular}{|c|c|c|c|}
\hline Factor & Cases with POAG $(\%)^{\mathrm{a}}$ & Controls $(\%)^{\mathrm{b}}$ & P-value \\
\hline \multicolumn{4}{|l|}{ IL-6 } \\
\hline Extracellular matrix cells & 76.14 & 53.00 & 0.0065 \\
\hline Vascular endothelial cells & 85.42 & 36.00 & $<0.0001$ \\
\hline Trabecular endothelial cells & 55.14 & 24.33 & 0.0007 \\
\hline \multicolumn{4}{|l|}{ IL-1 $\beta$} \\
\hline Extracellular matrix cells & 64.00 & 39.66 & 0.0002 \\
\hline Vascular endothelial cells & 70.42 & 38.33 & 0.0001 \\
\hline Trabecular endothelial cells & 57.55 & 33.66 & 0.0040 \\
\hline \multicolumn{4}{|l|}{ TNF- $\alpha$} \\
\hline Extracellular matrix cells & 80.42 & 30.66 & $<0.0001$ \\
\hline Vascular endothelial cells & 60.43 & 41.00 & 0.0298 \\
\hline Trabecular endothelial cells & 66.57 & 35.33 & 0.0001 \\
\hline \multicolumn{4}{|l|}{ TGF- $\beta 1$} \\
\hline Extracellular matrix cells & 86.43 & 29.33 & $<0.0001$ \\
\hline Vascular endothelial cells & 81.57 & 31.33 & $<0.0001$ \\
\hline Trabecular endothelial cells & 73.28 & 40.00 & $<0.0001$ \\
\hline \multicolumn{4}{|l|}{ VEGF } \\
\hline Extracellular matrix cells & 85.57 & 30.33 & $<0.0001$ \\
\hline Vascular endothelial cells & 72.28 & 15.33 & $<0.0001$ \\
\hline Trabecular endothelial cells & 59.86 & 29.33 & $<0.0001$ \\
\hline
\end{tabular}

IL, interleukin; TNF- $\alpha$, tumor necrosis factor $\alpha$; TGF-1 $\beta$; tumor growth factor $1 \beta$; VEGF, vascular endothelial growth factor. ${ }^{a} \mathrm{n}=7 \mathrm{with}$ one specimen per patient. ${ }^{b} \mathrm{n}=4$ with one specimen per patient.

including the presence of lymphocytes or plasma cells in the TM and the iridocorneal angle were also visible.

The pattern of distribution of resident tissue macrophages close to vascular beds indicates a guardian role at the blood-tissue interface. It is known that uveal tract macrophages produce pro-inflammatory substances (including nitric oxide and cytokines) in conditions, such as acute anterior or endogenous posterior uveitis. This could be of particular significance in light of their close proximity to the blood-ocular barriers (12). Neovascularization, associated with infiltration of lymphocytes and melanocytes, was also observed in the TM. The initiation of immune responses is dependent on the presentation of antigens to T-helper lymphocytes. T cells are the predominant inflammatory cell type (13) in the TM.

Cells of all regions of the TM, have thick bundles of actin filaments along their basal cytoplasm that are oriented in parallel, indicated as attachment plaques of microfilament at the plasma membrane (Fig. 2). In POAG the oxidative stress may trigger degeneration in human TM, which affects the cytoskeleton and the adhesive properties in TM cells, leading to increased IOP. Elevated pressure induced the disruption of tight junctions, which may result in changes in the microenvironment and damage the TM cells, thus modifying the normal outflow of AH. The effects of elevated IOP may also influence cell adhesion to collagen beams, which may partially explain the presence of fewer TM cells in POAG patients when compared with normal subjects (14).
Immunohistochemical analysis. Sections of TM samples were exposed to primary/secondary antibodies developing a dark-brown (intense), yellow-brown (slight) or no immune staining. The expression levels of IL-6, IL-1 $\beta$, TNF- $\alpha$, TGF- $\beta 1$ and VEGF are shown in Table I. Immunoreactivity was specific since no immunostaining was obtained in the control sections incubated with each primary antibody absorbed with the specific peptide or with pre-immune serum (data not shown). It was revealed that IL-6 is markedly expressed in human trabecular meshwork (Fig. 3). The concentration of IL-6 in the TM of patients with POAG was significantly higher compared with that of the control subjects. Normally, the cells do not synthesize and secrete IL-6 unless they are stimulated by other cytokines or by certain physiological events. It has been demonstrated that a wide range of ocular tissues can produce IL-6 under pathological conditions, such as cytokine-stimulated vascular endothelial cells and vascular smooth muscle cells (15). Inflammatory cells, particularly mast cells, are known to be able to stimulate IL-6 secretion from leukocytes and human vascular endothelial cells in ischemic and inflammatory conditions. The present study demonstrated that expression of other inflammatory cytokines in the trabecular meshwork, including IL-1 $\beta$ (Fig. 5A and B) and TNF- $\alpha$ (Fig. 4), that are significant components of the pro-inflammatory response and intraocular inflammation. IL-1 $\beta$ systemically regulates the metabolic, immuno-inflammatory and reparative properties of tissues, and can be a 


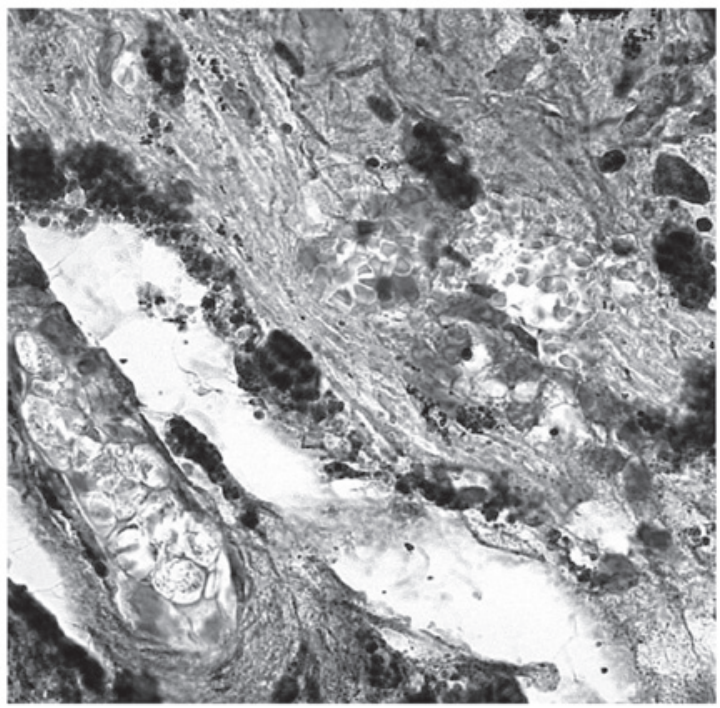

Figure 3. Micrograph of interleukin-6 immunostaining in human trabecular meshwork. Evident immunoreactivity was found in vascular endothelial cells (dark precipitates). Magnification, x40.

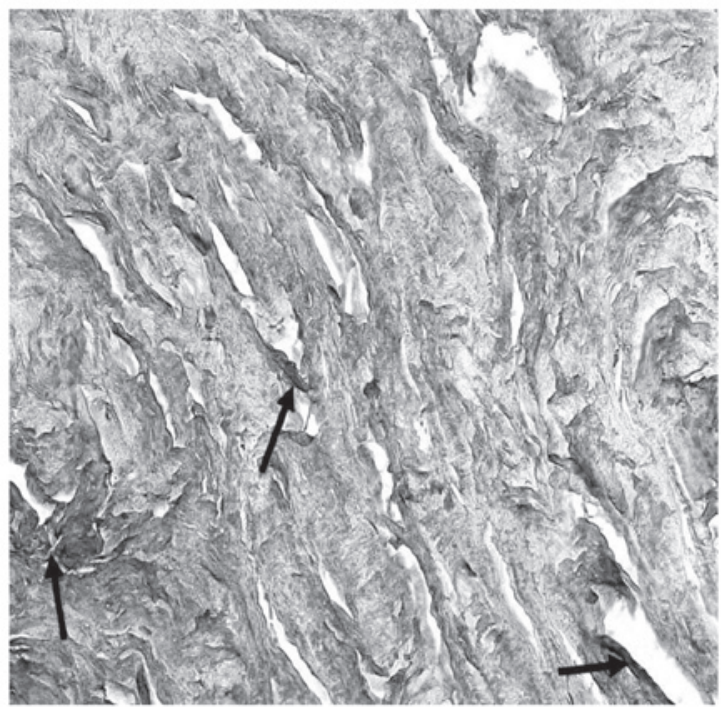

Figure 4. Micrograph of tumor necrosis factor- $\alpha$ immunostaining in human trabecular meshwork. Marked immunoreactivity (arrows) was observed around the vessels and in the vessel wall. Magnification, x20.

mediator of a number of diseases. It is secreted by monocytes and tissue macrophages, and is involved in the activation of $\mathrm{T}$ cells. In human TM tissues, evident immunoreactivity for IL-1 $\beta$ was observed in the macrophages of all the patients (all specimens) and moderate immunoreactivity was present in the blood vessels. Evident immunoreactivity for TNF- $\alpha$ was observed in uveal meshwork, particularly in the macrophages and lymphocytes of the samples analyzed. TNF- $\alpha$ is a cytokine secreted by lymphocytes and reticuloendothelial cells in numerous acute and chronic inflammatory diseases. TNF- $\alpha$, like IL-6, is also a pro-inflammatory cytokine and is involved in the blood-retinal barrier breakdown by opening tight junctions of retinal vascular endothelial cells (16). In the majority of the eyes, TGF- $\beta 1$ immunolabeling was predominantly observed in extracellular areas of the juxtacanalicular
A

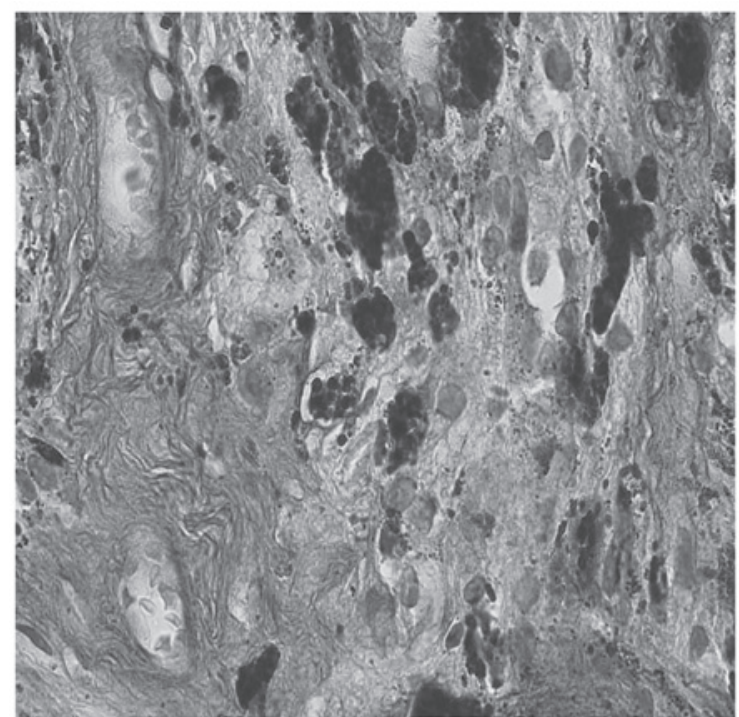

B

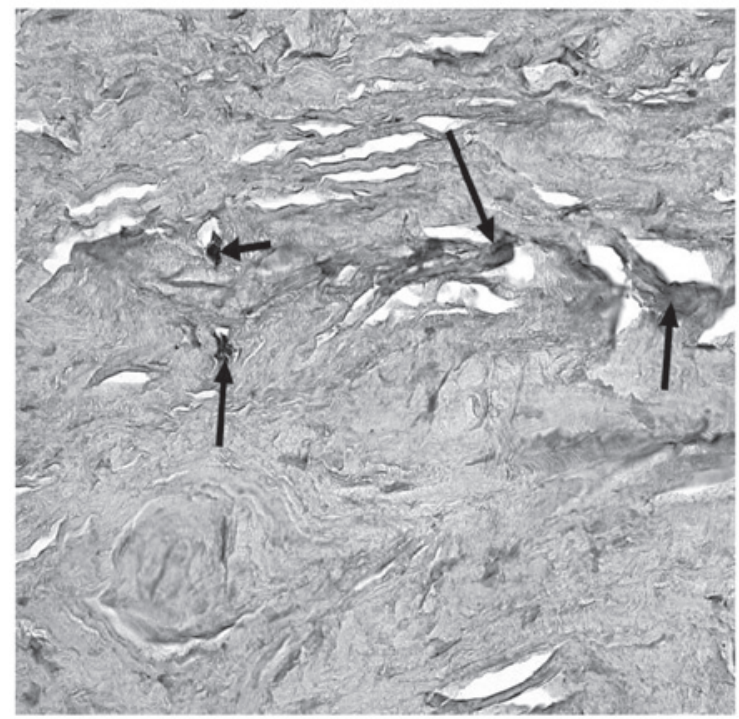

Figure 5. Micrograph of interleukin-1 $\beta$ immunostaining in human trabecular meshwork. Evident immunoreactivity (dark precipitates indicated by arrows) was found in the macrophages and in the blood vessels. (A) Magnification, x40; (B) magnification, x20.

(cribriform) section of the TM. An extent of focal staining was observed in the corneoscleral and uveal regions of the TM. In the eyes of six glaucoma patients, TGF- $\beta 1$ immunoreactivity was considerably more intense and all the regions of the TM were positively labelled (Fig. 6A and B). In human TM, VEGF revealed a moderate expression in extracellular areas of the juxtacanalicular (cribriform) region, while evident immunoreactivity was observed in the iris, in vessel endothelial cells and in fibroblasts (Fig. 7).

\section{Discussion}

In POAG patients, sustained increases in the trabecular outflow resistance commonly result in elevated IOP. Elevated IOP is the primary risk factor for glaucomatous optic neuropathy, which is associated with progressive vision loss. The molecular changes that lead to an increase in outflow resistance in POAG have not been clarified, and one hypothesis is based on the observation that the exposure of trabecular cells to mechanical stress, 
A

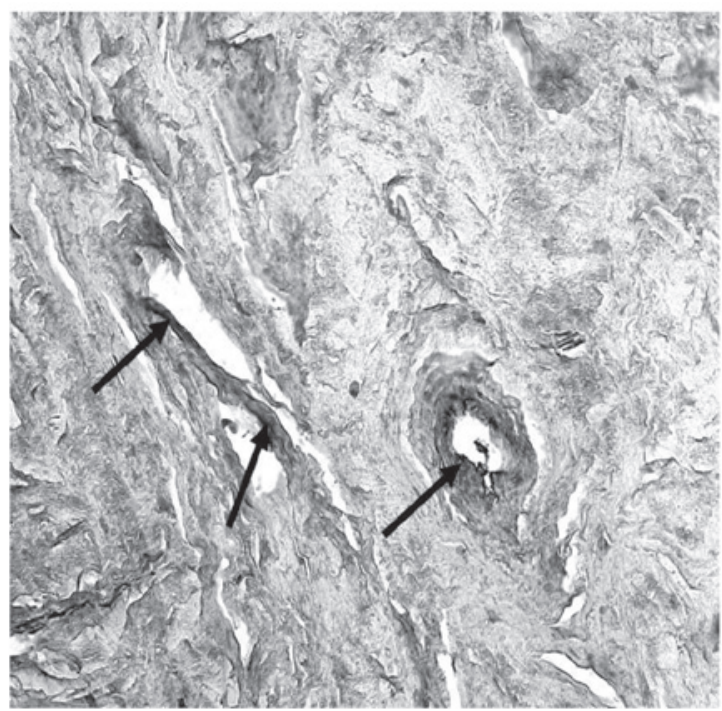

B

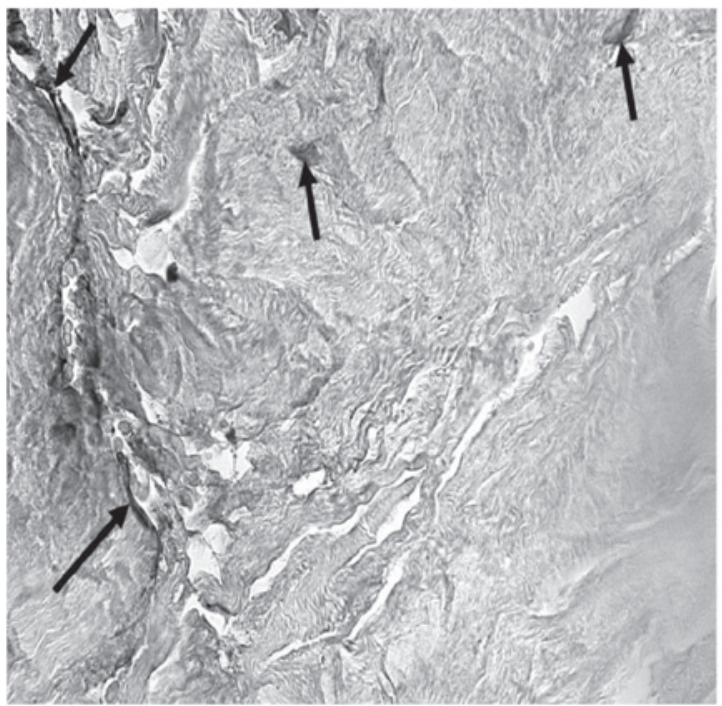

Figure 6. Micrograph of TGF- $\beta 1$ immunostaining in the human trabecular meshwork. Evident immunoreactivity for TGF- $\beta 1$ is observed in the wall of the vessels (indicated by arrows) of the TM (A) and in the extracellular compartment (B). Magnification, $x 20$. TGF- $\beta 1$, tumor growth factor- $\beta 1$; TM, trabecular meshwork.

caused by ocular vascular changes, increases the expression of TGF- $\beta 1$ in the TM. The overexpression of TGF- $\beta 1$ induces proliferation of fibrotic tissue, which determines the remodelling of the ECM, trabecular obstruction and increase in IOP (17). In patients with POAG, TGF- $\beta 1$ is widely expressed in the TM, in the $\mathrm{AH}$, inside the anterior chamber, in the neural retina and in retinal pigments. The increase of TGF- $\beta 1$ is due to the loss of the blood-eye barrier, thus the concentration of plasma-derived TGF- $\beta 1$ increases in the plasma, contributing to the high levels in the eye. TGF- $\beta 1$ reduces the production of enzymes that are responsible for the degradation of the ECM, is involved in fibrosis and induces the expression of various components of the ECM, including collagen type I, III, IV and VI, elastin, fibronectin and miociclin. Furthermore, TGF- $\beta 1$ increases the expression of transglutaminase, which induces irreversible cross-linking of fibronectin in trabecular bone. Tissue transglutaminase is normally expressed in trabecular cells and is found at high levels in patients with POAG (7).

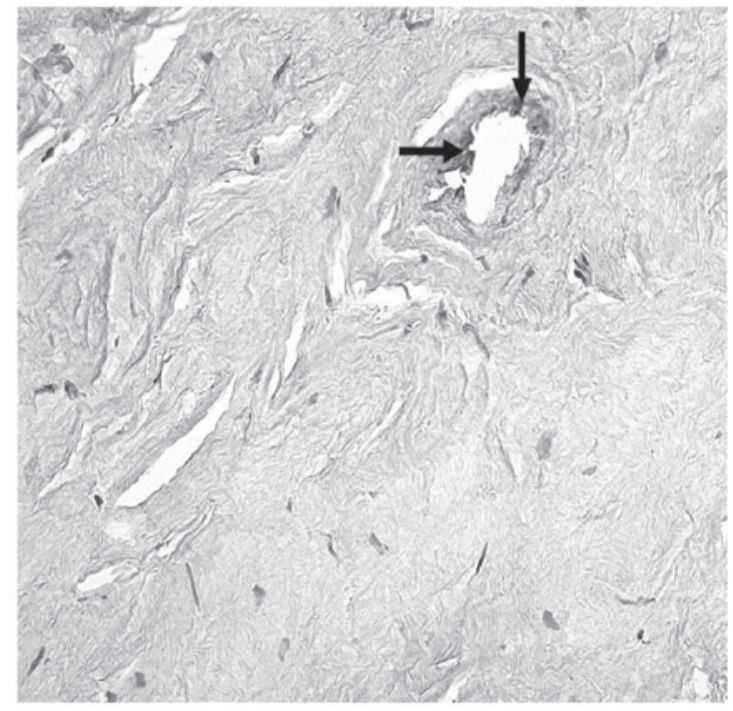

Figure 7. Micrograph of vascular endothelial growth factor immunostaining in human trabecular meshwork. Evident immunoreactivity is observed in the vessel wall (indicated by arrows). Magnification, x40.

TGF- $\beta 1$ has been demonstrated to promote transdifferentiation of fibroblasts into myofibroblasts that exhibit contractile activity and increase the rigidity of the TM (7).

The trabecular cells activate TGF- $\beta 1$ cross-orientation proteolysis or via trompospondin-1, which is a potent activator of TGF- $\beta 1$ in vivo and in vitro. The involvement of TGF- $\beta 1$ in the remodelling of the ECM and inflammation of glaucomatous eyes renders this protein a novel target for the treatment of glaucoma (17). In addition, TGF- $\beta 1$ regulates and induces the expression of IL-6 in the TM (18).

TM endothelial cells secrete a number of factors and cytokines that modulate the functions of the cells and the ECM of the conventional outflow pathway. In the TM the macrophages produce cytokines, such as IL- 6 , IL-1 $\beta$ and TNF- $\alpha$, leading to an acute inflammatory response and recruitment of other immune cells, including $\mathrm{T}$ lymphocytes. T-helper cells produce Th17 stimulated by IL-1 $\beta$, IL- 6 and TGF- $\beta 1$.

Increased levels of IL- 6 , IL- $1 \beta$ and TNF- $\alpha$ were observed in patients with POAG when compared with the control subjects, indicating that these cytokines may be correlated with disease activity. The continuous activation of macrophages and production of TNF- $\alpha$, IL- 6 and IL- $1 \beta$ prolongs the inflammatory response with production of large quantities of Th17. In fact, IL- 6 and Il-1 $\beta$ have been implicated in the differentiation of Th cells into Th17, which produce Il-17, and appear to be responsible for the transformation from acute to chronic inflammatory responses. In a normal patient, activation of fibroblasts, resulting in deposition of ECM, leads to a downregulation of the inflammatory reaction with transformation into a fibrotic reaction.

Pro-inflammatory cytokines, such as IL- 6 and TNF- $\alpha$, which are significant in inflammation, were detected at elevated levels in the AH of patients with neovascular glaucoma. In the neovascularization processes, there is a correlation between IL-6 and VEGF. IL-6 indirectly induces angiogenesis through stimulation of the expression of VEGF. A previous study revealed that interactions between fibroblasts, myofibroblasts 
and vascular endothelial cells occur at the site of the inflammatory lesion (11).

VEGF not only induces vascular proliferation, but also acts as a mediator in the process that leads to fibroblast proliferation. As angiogenesis is fundamental in the formation of granulation tissue (8), inhibition of neovascularization induced by anti-VEGF agents may decrease fibroblastic proliferation (19). The pathogenesis of these disorders is a result of a neuroinflammatory process (20). Pro-inflammatory cytokines and oxidative stress contribute to glaucomatous degeneration (21).

TNF- $\alpha$ is a pro-inflammatory cytokine secreted in response to infection and trauma. TNF- $\alpha$ is involved in the neurodegenerative process of glaucoma. In fact, this cytokine mediates the cytotoxic effect of ocular hypertension through a mechanism that involves activation of microglia and loss of oligodendrocytes.

The increase in IOP determines increased levels of TNF- $\alpha$, together with a massive expansion of the macrophage population. It is possible that the elevated IOP, with consequent hypoxia, could be responsible for the loss of the ocular blood-brain barrier, thus provoking an evident inflammatory response (22).

In conclusion, the present results add support to the evidence that growth factors and cytokines can induce ECM remodelling and alter cytoskeletal interactions in the TM.

The changes described may aid in the treatment of POAG. The generalized edema of the trabecular endothelium and the presence of inflammatory cells in POAG eyes, associated with an acute and marked rise of IOP could possibly respond better to a combination of anti-inflammatory therapy and glaucoma therapy. The gradual, chronic rise of IOP, is most likely due to the progressive activation of the inflammatory response, gradually transformed into a long-term fibrotic reaction. Therapy should therefore include anti-inflammatory drugs administered topically and systemically together with IOP-lowering agents, in order to control the inflammatory cells and reduce trabecular edema.

\section{References}

1. Resnikoff S, Pascolini D, Etya'ale D, Kocur I, Pararajasegaram R, Pokharel GP and Mariotti SP: Global data on visual impairment in the year 2002. Bull World Health Organ 82: 844-851, 2004.

2. Kwon YH, Fingert JH, Kuehn MH and Alward WL: Primary open-angle glaucoma. N Engl J Med 360: 1113-1124, 2009.

3. Quigley HA: Glaucoma. Lancet 377: 1367-1377, 2011.

4. Tamm ER, Toris CB, Crowston JG, Sit A, Lim S, Lambrou G and Alm A: Basic science of intraocular pressure. In: Intraocular Pressure. Reports and Consensus Statements of the 4th Global AIGS Consensus Meeting on Intraocular Pressure. Weinreb RN, Brandt JD, Garway-Heath D and Medeiros F (eds). Kugler Publications, Amsterdam, pp1-14, 2007.
5. Tamm ER: The trabecular meshwork outflow pathways: structural and functional aspects. Exp Eye Res 88: 648-655, 2009.

6. Barany EH: In vitro studies on the resistance to flow through the angle of the anterior chamber. Acta Soc Med Ups 59: 260-276, 1954

7. Fuchshofer R and Tamm ER: The role of TGF- $\beta$ in the pathogenesis of primary open-angle glaucoma. Cell Tissue Res 347: 279-290, 2012

8. Takeuchi K, Nakazawa M and Ebina Y: Effects of trehalose on VEGF-stimulated angiogenesis and myofibroblasts proliferation: implications for glaucoma filtration surgery. Invest Ophthalmol Vis Sci 52: 6987-6993, 2011.

9. Bianchi E, Scarinci F, Grande C, Plateroti R, Plateroti P, Plateroti AM, Fumagalli L, Capozzi P, Feher J and Artico M: Immunohistochemical profile of VEGF, TGF- $\beta$ and PGE $_{2}$ in human pterygium and normal conjunctiva: experimental study and review of the literature. Int J Immunopathol Pharm acol 25: 607-615, 2012

10. Cohen T, Nahari D, Cerem LW, Neufeld G and Levi BZ: Interleukin 6 induces the expression of vascular endothelial growth factor. J Biol Chem 271: 736-741, 1996.

11. Chen KH, Wu CC, Roy S, Lee SM and Liu JH: Increased interleukin-6 in aqueous humour of neovascular glaucoma. Invest Ophthalmol Vis Sci 40: 2627-2632, 1999.

12. McMenamin PG: The distribution of immune cells in the uveal tract of the normal eye. Eye (Lond) 11: 183-193, 1997.

13. Romeike A, Brügmann M and Drommer W: Immunohistochemical studies in equine recurrent uveitis (ERU). Vet Pathol 35: 515-526, 1998.

14. Yang X, Liu B, Bai Y, Chen M, Li Y, Chen M, Wei Y, Ge J and Zhuo Y: Elevated pressure downregulates ZO-1 expression and disrupts cytoskeleton and focal adhesion in human trabecular meshwork cells. Mol Vis 17: 2978-2985, 2011.

15. Yan SF, Tritto I, Pinsky D, et al: Induction of interleukin-6 (IL-6) by hypoxia in vascular cells. Central role of the binding site for nuclear factor-IL-6. J Biol Chem 270: 11463-11471, 1995.

16. Evans CA, Jellis J, Hughes SP, Remick DG and Friedland JS: Tumour necrosis factor-alpha, interleukin-6, and interleukin- 8 secretion and the acute-phase response in patients with bacterial and tuberculous osteomyelitis. J Infect Dis 177: 1582-1587, 1998.

17. Prendes MA, Harris A, Wirostko BR, Gerber AL and Siesky B: The role of transforming growth factor $\beta$ in glaucoma and the therapeutic implications. Br J Ophthalmol 97: 680-686, 2013.

18. Liton PB, Li G, Luna C, Gonzalez P and Epstein DL: Cross-talk between TGF-beta1 and IL-6 in human trabecular meshwork cells. Mol Vis 15: 326-334, 2009.

19. Horsley MB and Kahook MY: Anti-VEGF therapy for glaucoma. Curr Opin Ophthalmol 21: 112-117, 2010.

20. Ahmed F, Brown KM, Stephan DA, Morrison JC, Johson EC and Tomarev SI: Microarray analysis of changes in mRNA levels in the rat retina after experimental elevation of intraocular pressure. Invest Ophthalmol Vis Sci 45: 1247-1258, 2004.

21. Tezel G: Oxidative stress in glaucomatous neurodegeneration: mechanisms and consequences. Prog Retin Eye Res 25: 490-513, 2006

22. Roh M, Zhang Y, Murakami Y, Thanos A, Lee SC, Vavvas DG, Benowitz LI and Miller JW: Etanercept, a widely used inhibitor of tumor necrosis factor- $\alpha$ (TNF- $\alpha$ ), prevents retinal ganglion cell loss in a rat model of glaucoma. PLoS One 7: e40065, 2012. 